\title{
Thermography measurements and latent heat documentation of Norwegian spruce (Picea abies) exposed to dynamic indoor climate
}

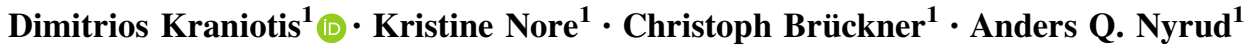

Received: 22 June 2015/Accepted: 5 November 2015/Published online: 8 December 2015

(C) The Author(s) 2015. This article is published with open access at Springerlink.com

\begin{abstract}
Hygroscopic materials hold the potential to reduce ventilation loads in a building by damping the peak fluctuations of the indoor relative humidity. Of particular interest is the moisture buffer capacity of wooden surfaces. This paper investigates, the response of Norwegian spruce (Picea abies) samples to changes of indoor relative humidity as well as the corresponding latent heat release during moisture buffering. A climate chamber was used to subject samples to dynamic air humidity. Thermographic techniques and thermocouples were employed to measure surface temperature on the exposed surface of the spruce sample (permeable case), while a covered control sample was used as reference (impermeable case). The moisture uptake was logged synchronously by weighting cells. The results show that the surface temperature of spruce increased by $2.1{ }^{\circ} \mathrm{C}$ during moisture uptake, while only by $0.9^{\circ} \mathrm{C}$ in the control sample. This finding has implications for direct energy savings when wooden surfaces are used indoors. In addition, thermography is evaluated as an appropriate measurement method for documenting latent heat release.
\end{abstract}

Dimitrios Kraniotis

dimitrios.kraniotis@treteknisk.no

Kristine Nore

kristine.nore@treteknisk.no

Christoph Brückner

christoph.brueckner@treteknisk.no

Anders Q. Nyrud

anders.nyrud@treteknisk.no

1 Norwegian Institute of Wood Technology, Forskningsveien 3B, 0373 Oslo, Norway
Keywords Latent heat - Hygroscopic material . Norwegian spruce (Picea abies) - Surface physics . Thermography

\section{Introduction}

Awareness of the influence of hygroscopic materials on reducing ventilation loads in buildings-and thus saving energy - is growing. The moisture-buffering capacity of hygroscopic materials, has been analyzed in several studies [1-4]. Recently, a new material property, the so-called 'moisture buffer value' (MBV), was developed [5] and describes the ability of building materials and systems of materials to exchange moisture with the indoor environment. The MBV of wood is triple that of concrete and brick, double that of gypsum and about $20 \%$ higher than cellular concrete [5]. Rode and Grau [3] showed that hygroscopic wall surfaces provide a noticeable effect on damping of indoor humidity variations; the higher the MBV of a material the greater the damping.

The contribution of moisture buffer capacity of wooden surfaces on damping the relative humidity $(\mathrm{RH})$ has been reported [e.g., 3, 6]. Simonson et al. [1] showed that when the interior surfaces of a wooden apartment building were permeable, the maximum indoor RH was lower as compared to the impermeable case (impermeable paint). In addition, the RH dropped below $20 \%$ for less time as compared to the impermeable case. Furthermore, Osanyintola and Simonson [2] showed that using hygroscopic materials, such as wood, combined with a well-controlled heating, ventilation and air conditioning (HVAC) system, the potential for direct energy savings is relatively small for heating, i.e., $2-3 \%$ of the total heating energy, but significant for cooling, i.e., 5-30\% of the total cooling 
energy. The potential indirect savings from adjusting the ventilation rate and indoor temperature, while maintaining adequate indoor air quality and comfort, are in the order of $5 \%$ for heating while they range from 5 to $20 \%$ for cooling. Woloszyn et al. [4] confirmed that the use of gypsum-based moisture-buffering materials, combined with a relative humidity sensitive (RHS) ventilation system, could reduce the mean ventilation rate by $30-40 \%$ and generate 12-17\% energy savings while during the heating period. The combined effect of ventilation and wood, as buffering material, make it possible to maintain a stable indoor RH between 43 and $59 \%$.

Mendes et al. [7] used a heat and mass transfer model to show the effects of moisture on sensible and latent conduction loads. The results showed that excluding moisture transport in the building envelope from the whole-building energy simulation models may result in overestimation of conduction peak loads, but also in underestimation of the yearly integrated heat fluxes. The latter may lead to over dimensioning the HVAC, especially in dry climates, as well as to underestimate energy consumption, primarily in humid climates.

The fact that hygroscopic materials can moderate indoor humidity is important because it shows that the moisture produced in a space is not directly transferred to the ventilated air as assumed in current ventilations design methods. Since heat and moisture transfer are coupled, the indoor temperature will increase when moisture accumulates in the hygroscopic structure and decrease when moisture evaporates from the structure.

A hygrothermal model accounting for moisture and heat transport in a solid wood envelope directly exposed to an indoor climate has been presented by Hameury [8]. The model incorporates the moisture diffusion process through a wood structure. The results show that the migration of moisture through the wood layer of a solid timber building envelope generates heat which leads to an increase or decrease of the wood temperature.

Latent heat is the amount of heat required for the phase change of a substance without any temperature fluctuations. When condensation occurs from a vapor phase to a liquid phase, heat is released at a rate of $2501 \mathrm{~kJ} / \mathrm{kg}$ of vapor condensing at $0{ }^{\circ} \mathrm{C}$. This is the latent heat of vaporization of water, $H_{\mathrm{v}}$. At typical indoor temperatures, i.e., $10-30{ }^{\circ} \mathrm{C}, H_{\mathrm{v}}$ decreases from 2477.7 to $2430.5 \mathrm{~kJ} / \mathrm{kg}$ [8]. The enthalpy of sorbed water is less than that of liquid water and the differential heat of sorption $\Delta H_{\mathrm{s}}$ has to be added to the latent heat of vaporization of water in order to equal the total latent heat of sorption $H_{\mathrm{m}}$ of bound water in the cell walls of a wood structure. Thus, the latent heat of moisture $H_{\mathrm{m}}$ is the sum of the latent heat of vaporization of water $H_{\mathrm{v}}$ and the differential heat of sorption $\Delta H_{\mathrm{s}}$, described by the equation [8]:
$H_{\mathrm{m}}=H_{\mathrm{v}}+\Delta H_{\mathrm{s}}$

Even though relevant studies have displayed the advantageous role of hygroscopic materials by defining their hygrothermal behavior, the energy potential is not taken into account. This raises an important question; is it possible to take advantage of the effect of latent heat in order to save energy? Porous surfaces and large inner surface area highly interacts with the moisture loads of indoor environment, by absorption and desorption.

The objective of this study is to document and discuss the energy (radiation) emitted by untreated wood, in particular Norwegian spruce (Picea abies), when changes in the indoor RH occur. The scenarios of an exposed-permeable sample and a covered-impermeable sample are studied. The increase of the indoor RH is shown in relation to water uptake of the two samples. The radiation emitted by the two samples is recorded using thermography techniques and their respective surface temperature is calculated. By comparing to results obtained from thermocouples, the suitability of thermography as a technique for observing latent heat release is evaluated. The weight uptake of spruce has been also recorded and is correlated to the surface temperature time series.

\section{Materials and methods}

The experiment took place in a $41 \mathrm{~m}^{3}$ dark climate chamber with ceiling-mounted ventilation. Alternating $\mathrm{RH}$ was defined with constant temperature and air velocity. The sample housing was built of medium-density fibreboard (MDF) covered in aluminium foil. A $0.5 \mathrm{~m}^{2}$ area on the ceiling of the chamber was found for optimal placing with an average air velocity of $0.15 \pm 0.05 \mathrm{~m} / \mathrm{s}$ according to the air speed meter.

A series of pre-tests were carried out, showing vertical variations in surface temperature because of thermal buoyancy forces when the samples were wall-mounted. To avoid this source of error, the samples were ceilingmounted. Except for the thermographic camera and the PClogger, all electrical instruments were installed outside the room to minimise signal noise.

The samples used in the experiments were spruce heartwood. The left-mounted sample was covered with transparent low-density polyethylene (LDPE) foil which is water repellent (simulating an impermeable surface), hereinafter called the reference sample. The right-mounted sample was exposed, simulating a permeable surface, hereinafter called the spruce sample (Fig. 1). Five sides of the samples were covered with adhesive tape and LDPE preventing moisture uptake, while the open side faced the room. The two samples had the same number of annual 
Fig. 1 a Housing, samples (covered left, exposed right) and thermocouples mounted on the ceiling. The air speed meter was placed in the centre (photo taken from the position of the thermographic camera). b Sketch-section of the apparatus with the samples and the weight cells
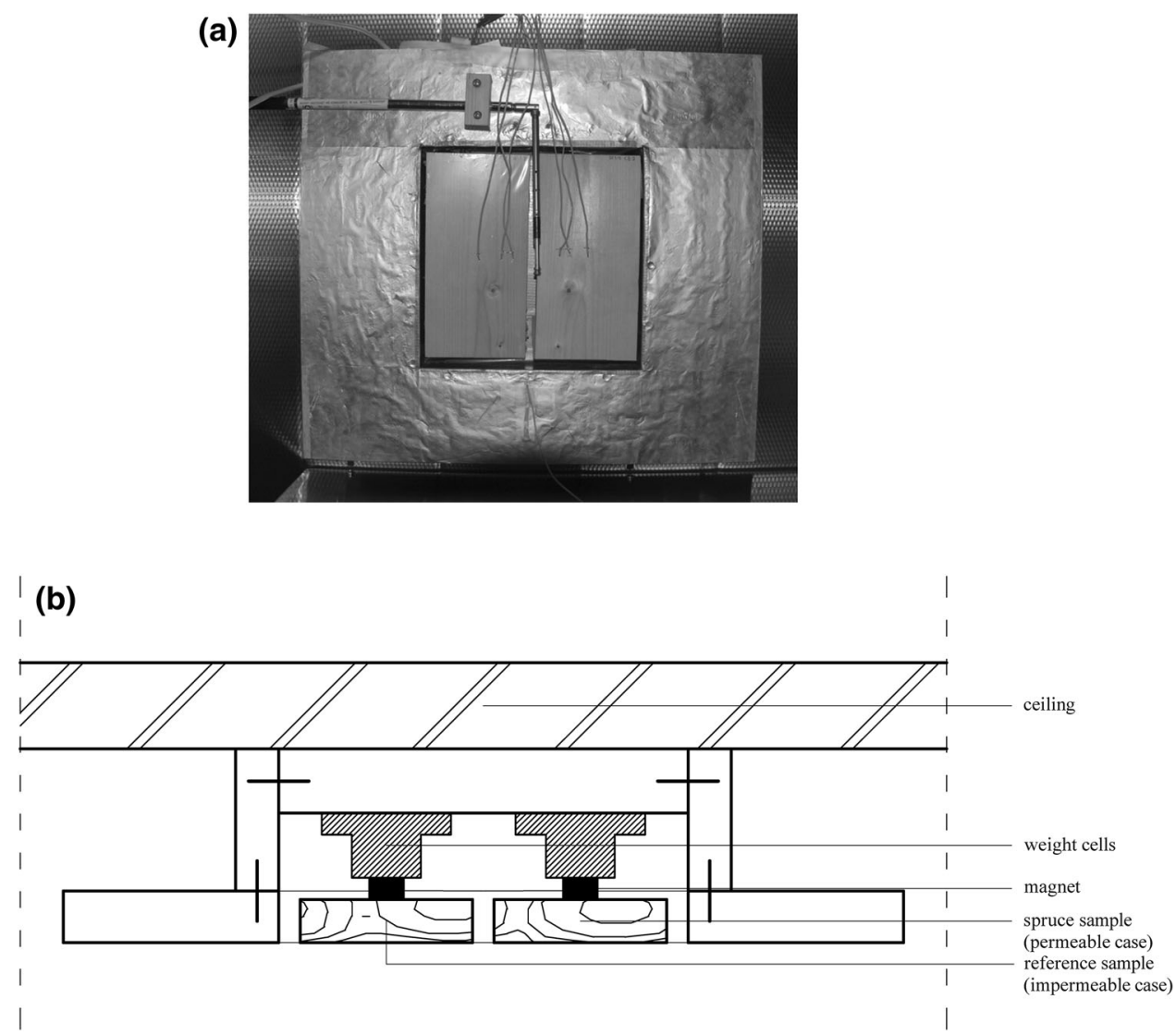

rings. The samples were $200 \mathrm{~mm}$ long by $100 \mathrm{~mm}$ wide and $10 \mathrm{~mm}$ thick. The spruce sample and the reference sample were sliced from the same panel piece and were mirrored in order to have equal surface texture.

Thermography techniques were employed in order to measure radiation emitted by the monitoring surfaces and consequently to calculate the corresponding surface temperature based on the Stefan-Boltzmann law:

$T=\sqrt[4]{\varepsilon \times \sigma / W_{\mathrm{RB}}}$

where $W_{\mathrm{RB}}$ is the radiation emitted by a real body, $\varepsilon$ is the emissivity and $\sigma$ is the Stefan-Boltzmann constant $\left(\sigma=5.67 \times 10^{-8} \mathrm{Wm}^{-2} \mathrm{~K}^{-4}\right)$. Emissivity is the ability of a surface to radiate energy. The value of emissivity for a real body is described as the ratio of the radiant flux emitted per unit area to that emitted by a black body at the same temperature [9].

Another important quantity for thermographic techniques is the transmittance of a material. It is defined as the proportion of infrared radiant energy impinging on an object's surface that is transmitted through the object. For the common opaque materials the value of transmittance (transmissivity) is $\tau=0$.

In general, all real bodies have the properties to absorb radiation. The induced radiation will be absorbed, transmitted or reflected, expressed by the equation: $\alpha+\rho+\tau=1$

where $\alpha$ is the absorptivity, $\rho$ is the reflectivity and $\tau$ is the transmissivity of a real body.

According to Kirchhoff's law, the ability of every object to absorb incoming radiation energy equals its ability to emit energy at the same wavelengths, i.e., $\alpha=\varepsilon$. Thus, Eq. 3 can be written as

$\varepsilon+\rho+\tau=1$

The transmissivity of the LDPE foil was measured, $\tau_{\mathrm{LDPE}}=0.835$. The emissivity of the exposed spruce sample was measured, $\varepsilon_{\mathrm{sp}}=0.90$. The coupled emissivity of the foil and the wood sample, as one object, is not equal to the emissivity of the exposed wood sample $\varepsilon_{\mathrm{sp}}$, but needs to be corrected by the factor of transmittance of the LDPE foil that covers it. Thus, the emissivity of the covered sample $\varepsilon_{\mathrm{cov}}$ (reference) was calculated, $\varepsilon=0.75$, based on the following simplified equation:

$\varepsilon_{\mathrm{cov}}=\varepsilon_{\mathrm{sp}} \times \tau_{\mathrm{LDPE}}$

The reason that the sample housing was covered in aluminium foil is that aluminium has a very low emissivity $(\varepsilon=0.03)$ and hardly contributes to undesired reflections on the measured target, i.e., samples, when taking thermographic images [9]. 
A thermographic camera, placed at a distance of $0.5 \mathrm{~m}$, recorded the experiment. A relevant software allowed plotting average temperature of the monitoring areas of the two samples. Three wired thermocouples of type $\mathrm{K}$ were stapled to each sample. The sensors were pressed halfway in the wood and the LDPE foil to measure the surface temperature. Each sample was held by a weighting cell, which measured its mass change. The surface temperature and weight of each sample was recorded every $5 \mathrm{~s}$. Room temperature and average $\mathrm{RH}$ were logged every minute by the climatic chamber sensors situated close to the inlet fan. Average air velocity and temperature, $10 \mathrm{~mm}$ below the monitored surfaces, were measured every $20 \mathrm{~min}$ by an air velocity meter placed halfway between sample and reference, see Fig. 1.

In order to obtain conditions of equilibrium for the samples, a preliminary test was performed before the main experiment. The samples were placed in the climate chamber and the RH of the room was adjusted to $25 \%$. The RH in the room was increased to $90 \%$ over a $12 \mathrm{~h}$ period, then decreased back to $25 \%$ over the next $12 \mathrm{~h}$. The intent of this one-day preliminary test was to obtain uniform surface temperature and equilibrium moisture content (EMC) of about $19 \%$ in the samples. Having ensured that the equilibrium conditions had been reached, the main experiment started. During the 12-h experiment period, the $\mathrm{RH}$ of the climate chamber was set to increase from 25 to $90 \%$. The temperature of the chamber was set at $19{ }^{\circ} \mathrm{C}$ resulting in the desired air temperature of approximately $20^{\circ} \mathrm{C}$, due to stratification, near the samples at the ceiling. The results from the main experiment are presented and discussed in the following section.

\section{Results and discussion}

The room temperature and the indoor $\mathrm{RH}$ for a period of 20 min are shown in Fig. 2. As seen, the moisture load was slightly higher than programmed. The RH in the room increased from $21.6 \%$ to a peak of $93.5 \%$ within the first $5 \mathrm{~min}$ and after $15 \mathrm{~min}$ it stabilised at $90 \%$ (Fig. 2). A temperature increased from 19.0 to $21^{\circ} \mathrm{C}$, during the change of $\mathrm{RH}$, was most likely caused by the vaporizer.

During the experiment, the air velocity stayed constant at $0.15 \pm 0.015 \mathrm{~m} / \mathrm{s}$ and thus the influence of air velocity on moisture absorption was not investigated. The value did not exceed typical indoor air velocities of around $0.40 \mathrm{~m} / \mathrm{s}$ [10].

Figure 3 shows the time series of surface temperature for the spruce and reference samples during the first hour of the measuring period. The initial temperature conditions on

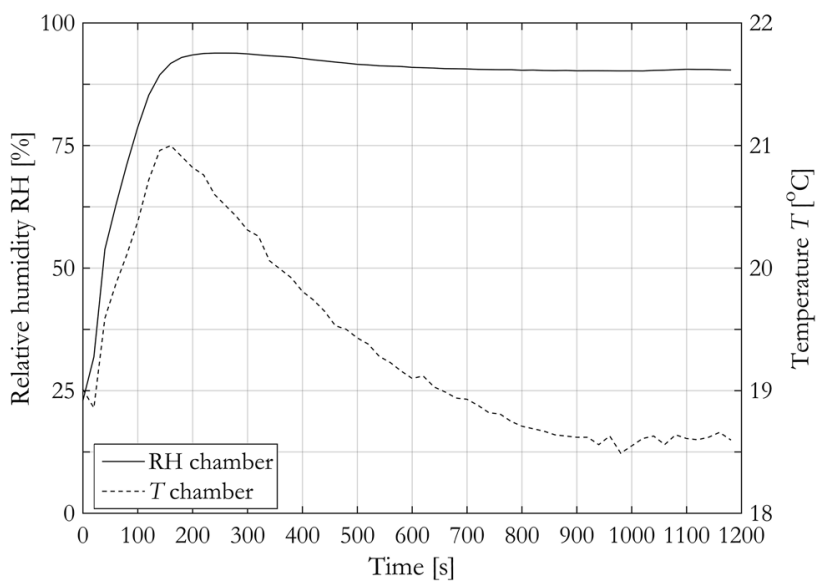

Fig. 2 Relative humidity and air temperature in the climate chamber

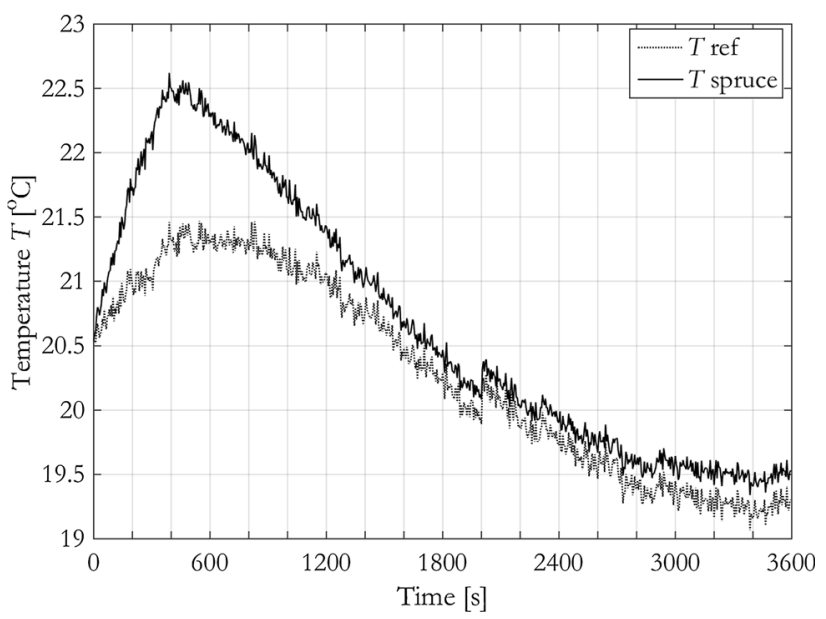

Fig. 3 Surface temperature on spruce and reference samples

the spruce sample's surface was $T_{\text {spruce }}=20.5{ }^{\circ} \mathrm{C}$. At $t=500 \mathrm{~s}, T_{\text {spruce }}$ had increased by $2.1{ }^{\circ} \mathrm{C}$ to the maximum of $22.6{ }^{\circ} \mathrm{C}$. Even at $t \approx 1200 \mathrm{~s}$, the surface of spruce was still heated by $0.8^{\circ} \mathrm{C}$.

The maximum temperature increase of the reference sample, also measured at $t=500 \mathrm{~s}$, was $\Delta T_{\text {ref }}=0.9{ }^{\circ} \mathrm{C}$ (Fig. 3). The temperature increase on the surface of the reference sample was due to the room temperature increase and vapor condensation on its surface.

The spruce sample showed an instant surface temperature change when the RH was changed. Over the first hour, the total weight gain of the spruce sample was $\Delta W_{\mathrm{u}}=11.7 \mathrm{~g}$, where $W_{\mathrm{u}}$ is the absolute weight of the samples (Fig. 4). The absorption rate was fastest initially, with a water uptake of approximately $7.5 \mathrm{~g}$ from $t=0 \mathrm{~s}$ to $t=500 \mathrm{~s}$, when $T_{\text {spruce,max }}$ happened. From $t=500 \mathrm{~s}$ until $t=1500 \mathrm{~s}$ water uptake continued to increase 


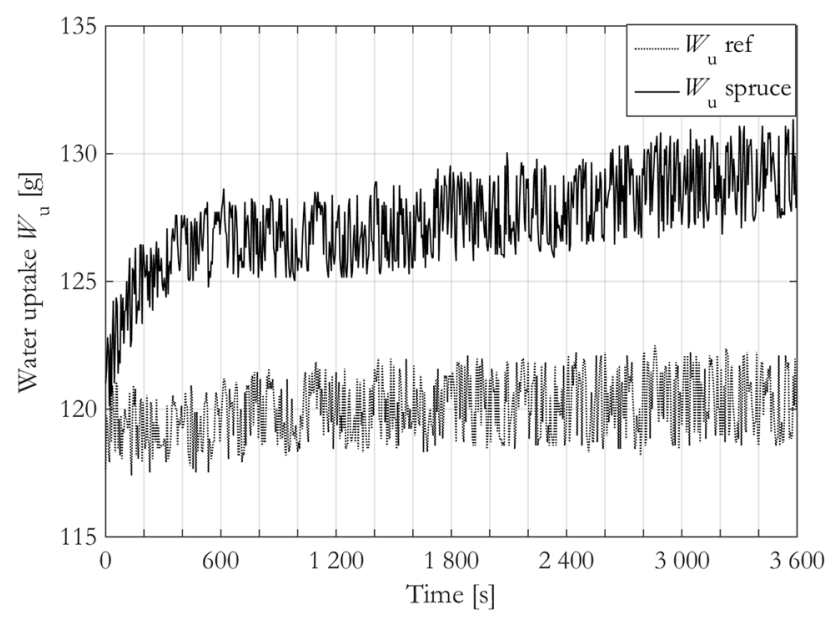

Fig. 4 Weight gain of spruce and reference samples

parabolically. After $1500 \mathrm{~s}$ water uptake increased linearly. The exposed spruce sample was reacted more in absorption as compared to the impermeable reference sample, which holds a key role for ongoing latent heat release. The reference sample gained weight caused by condensed water drops on the front and backside (Fig. 4). The fluctuations of the water uptake $W_{\mathrm{u}}$ in Fig. 4 for both the spruce and the reference sample reflects the tolerance of the weight cells, i.e., $\pm 2 \mathrm{~g}$.

Most of the absorption occurred during the beginning of the climate cycle, when thermography recorded the temperature increase (Fig. 5b). The spruce sample took up moisture and emitted heat. In contrast, the reference sample radiated less thermal energy (indicated by blue color) than the spruce sample (Fig. 5a). The camera's reflection on the reference sample is visible. The thermocouple results were less accurate than the thermography. In particular, the thermocouples measure the surface temperature punctually (in this study 3 points for each sample) so the surface temperature of the whole area of each of them is represented by the average of these three punctual measurements. In contrast, the thermal camera gives the surface temperature of an area as the average of $135 \times 68(=9180)$ measuring points, as many as the pixels of the picture captured by the camera. In addition, the thermocouples were half-mounted in the structure of the samples, thus they did not measure exactly the surface temperature as the thermal camera. The two variables, i.e., surface temperature measured by the thermocouples and the ones measured by the thermal camera, are related with a correlation coefficient of +0.9 during the observed time period $(1 \mathrm{~h})$.

\section{Conclusion}

This study investigated the latent heat release of a spruce surface during a change of interior relative humidity from 20 to $90 \%$. The results showed that the exposed spruce sample reacted drastically to the change of the RH in the climate chamber, resulting in an increase of the surface temperature. In contrast, the surface temperature increase of the reference sample, which was covered with transparent Low-Density Polyethylene foil, was significantly less. The surface temperature increase was correlated to the weight gain, and thus latent heat was also connected to moisture uptake of the spruce sample. The expectation of a higher surface temperature on the spruce sample due to vapor absorption was confirmed. The spruce sample (permeable case) functioned as a radiating panel when the $\mathrm{RH}$ of the room increased. In contrast, the surface temperature of the reference sample (impermeable case) increased much less.

In addition, this paper examined the relevance of thermographic techniques for measuring latent heat exchange. Thermography was shown to be an adequate method for measuring latent heat on surfaces, due to its high sensitivity to temperature changes. Using thermography made it possible to observe the surface heating, in real time, in response to increasing $\mathrm{RH}$.

The findings reveal a potential for direct heat gains emitted by untreated or vapor-diffusive wooden structures when vapors are absorbed in wood structures. The permeable wood surfaces function as radiating heating panels during water uptake. The latent heat process that describes the phenomena reveals that latent heat exchange can be an important fraction of the total energy balance that describes a room. In this context, latent heat ought to be taken into account when designing HVAC systems. The fast RH rise can represent e.g., rapid moisture excess in bathrooms (showering), while the slow RH-fall was adapted to daily drying in buildings while high temperatures outdoors. The authors wish to provide a base for strategies to use wood (a low-tech natural material) to reduce the need for energy. Future studies will examine different wood species at varying indoor climates; their effect on the energy balance and the correlation between latent heat exchange and wood properties.

Further experiments investigating surface warming through latent heat exchange are important for improving energy efficiency in the building industry. The measuring methods developed for this study establish a foundation 
Fig. 5 Thermographic image of reference sample (left) and spruce sample (right) during moisture uptake. a At $t=0 \mathrm{~s}$ and $\mathbf{b}$ at $t=500 \mathrm{~s}$. The camera's software provide the opportunity to include surface areas with different emissivity in the same picture
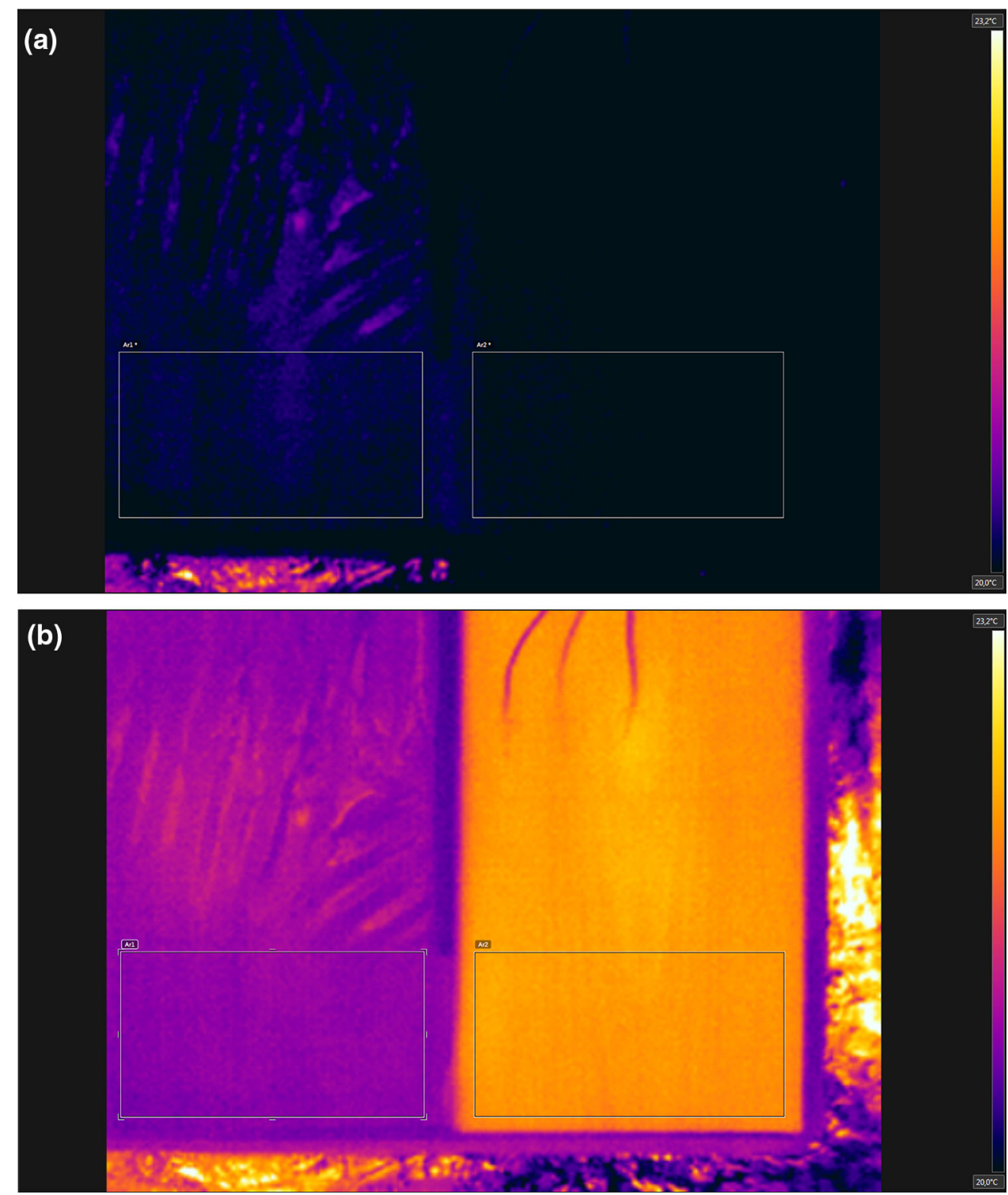

for further investigations on latent heat exchange on porous surfaces. The results also support future comparative climate simulations to validate building modelling software.

Acknowledgments The authors gratefully acknowledge the Norwegian Research Council (NRC) and industry taking part in the research project WEEE Wood-Energy, Emissions, Experience, No. 216404 , for funding this study.

Open Access This article is distributed under the terms of the Creative Commons Attribution 4.0 International License (http://crea tivecommons.org/licenses/by/4.0/), which permits unrestricted use, distribution, and reproduction in any medium, provided you give appropriate credit to the original author(s) and the source, provide a link to the Creative Commons license, and indicate if changes were made.

\section{References}

1. Simonson CJ, Salonvaara M, Ojanen T (2001) Improving indoor climate and comfort with wooden structures. VTT Publications 431, Technical Research Centre of Finland, Espoo

2. Osanyintola OF, Simonson CJ (2006) Moisture buffering capacity of hygroscopic building materials: experimental facilities and energy impact. Energy Build 38:1270-1282

3. Rode C, Grau K (2008) Moisture buffering and its consequence in whole building hygrothermal modeling. J Build Phys $31: 333-360$

4. Woloszyn M, Kalamees T, Abadie MO, Steeman M, Kalagasidis AS (2009) The effect of combining a relative-humidity-sensitive ventilation system with the moisture-buffering capacity of materials on indoor climate and energy efficiency of buildings. Build Environ 44:515-524

5. Rode C, Peuhkuri R, Mortensen LH, Hansen KK, Time B, Gustavsen A, Ojanen T, Ahonen J, Svennberg K, Harderup LE, 
Arfvidson J (2005) Moisture buffering of building materials. Project No.: 04023. Nordic Innovation Centre

6. Li Y, Fazio P, Rao J (2012) An investigation of moisture buffering performance of wood panelling at room level and its buffering effect on a test room. Build Environ 47:205-216

7. Mendes N, Winkelmann FC, Lamberts R, Philippi PC (2003) Moisture effects on conduction loads. Energy Build 35:631-644

8. Hameury S (2006) Moisture buffering capacity of heavy timber structures directly exposed to an indoor climate. A numerical study. Build Environ 40:1400-1412
9. Madding RP (1979) Thermographic instruments and systems. University of Wisconsin, Extension, Department of Engineering and Applied Sciences, Madison, Wisconsin

10. Toftum J (2004) Air movement—good or bad? International Centre for Indoor Environment and Energy, Technical University of Denmark, Lyngby 